\title{
Kinematic Properties of the TDG Candidates of CG J1720-67.8
}

\author{
Sonia Temporin \\ Institute of Astrophysics, University of Innsbruck, Austria
}

\begin{abstract}
I use integral field and long-slit spectra from the AAT and the Magellan telescope to investigate the kinematics of several clumps, recently identified along the prominent tidal tail of CG J1720-67.8 and suggested as possible tidal dwarf galaxy (TDG) candidates. A comparison of photometric and spectroscopic data with evolutionary synthesis models suggests burst ages of $\sim 6 \mathrm{Myr}$ for these clumps/TDG candidates.
\end{abstract}

\section{Introduction}

Tidal tails in interacting/merging systems are sites of super-star cluster and dwarf galaxy formation. The dense environment of compact galaxy groups offers an extraordinary chance to observe this phenomenon. Here I investigate the kinematics of the prominent tidal tail of the compact galaxy group CG J1720-67.8 through integral field spectra obtained at the Anglo-Australian Telescope (AAO) and a long-slit spectrum, kindly provided by F. Kerber, obtained at the Magellan telescope (LCO). This study complements a previous analysis (Temporin et al. 2003a,b) of the TDG candidates identified in this group.

\section{Properties of TDG candidates}

Kinematics and Dynamics- The prominent tidal tail of CG J1720-67.8 shows a general trend of decreasing radial velocity from north to south with a maximum velocity difference of $\sim 400 \mathrm{~km} \mathrm{~s}^{-1}$, as we found from descrete measurements of the clumps along it (Temporin et al. 2003a). The TDG candidate " $3+9$ " at the northern tip of the tail (Fig. 1a) shows a steeper velocity gradient (Fig. 1b): 150 to $200 \mathrm{~km} \mathrm{~s}^{-1}$ on a projected extent of $\sim 5 \mathrm{kpc}\left(\mathrm{H}_{0}=75 \mathrm{~km} \mathrm{~s}^{-1} \mathrm{Mpc}^{-1}\right)$. The long-slit spectrum taken across the southern/central part of the tail shows that beside the already observed general trend, there are local velocity gradients within individual TDG candidates (Fig. 1c). The lack of a continuous trend between adjacent clumps suggests that they might be kinematically distinct structures. The observed local velocity gradients, of order of $20 \mathrm{~km} \mathrm{~s}^{-1} \mathrm{kpc}^{-1}$, are comparable to those found in other TDGs (Mendes de Oliveira et al. 2001).

Evolutionary History - A previous analysis of optical colors indicated a young burst age for the TDG candidates, in the range 7 to $20 \mathrm{Myr}$ (Temporin et al. 2003b). By comparing the observations with SB99 models (Leitherer et al. 1999), I found a good agreement of optical/near-infrared colors and $\mathrm{H} \alpha$ 
equivalent widths with instantaneous burst models of age 5.5 to $8.5 \mathrm{Myr}$, once a correction for internal extinction was applied. Extinction-corrected spectra are in good agreement with model spectra of age $\sim 6 \mathrm{Myr}$ and total masses of order $10^{7} \mathrm{M}_{\odot}$ (Fig. 1d). All pieces of evidence suggest that these clumps have bursts much younger than their parent galaxies, for which ages of $\sim 40$ to $180 \mathrm{Myr}$ were estimated.
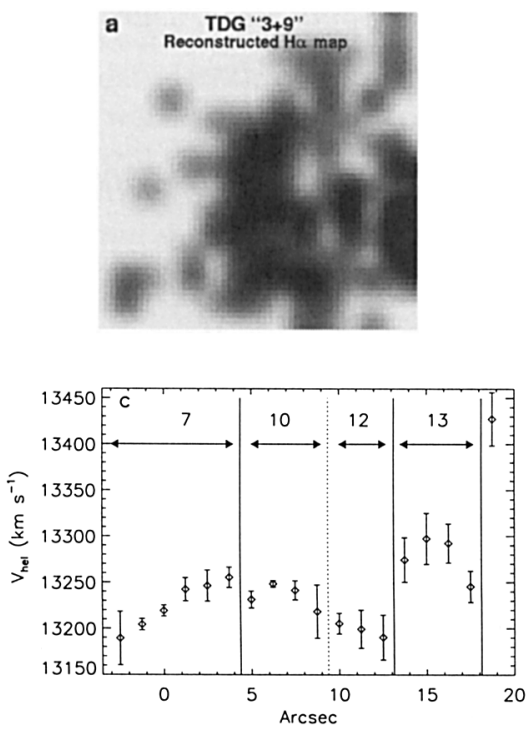
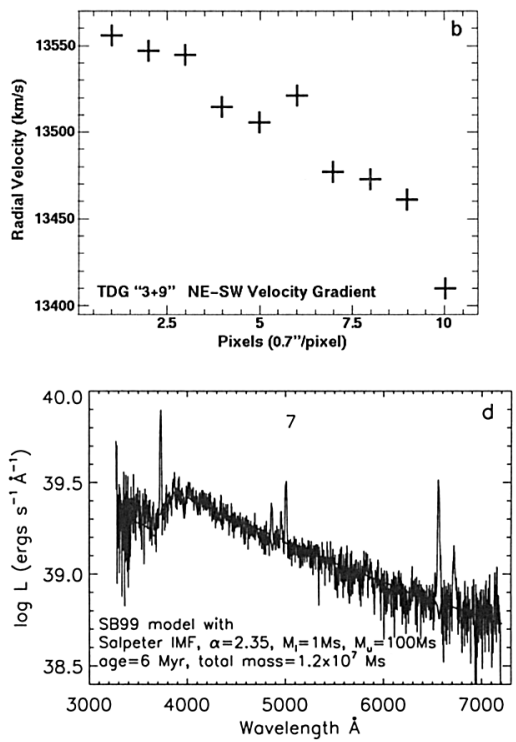

Figure 1. a) $\mathrm{H} \alpha$ map of the TDG " $3+9$ " reconstructed from integral field data. b) Radial velocity gradient across TDG " $3+9$ ". c) Velocity curve along the southern part of the tidal tail. Individual clumps are labeled. d) Spectrum of TDG "7" compared with an SB99 model, scaled in mass to match the observed, extinction-corrected luminosity.

In conclusion, the photometric and spectroscopic properties of these tidal objects are in agreement with what is expected for TDGs. Although projection effects might explain part of the observed local velocity gradients, kinematic data seem to indicate the presence of self-gravitation in the individual clumps.

Acknowledgments. I am grateful to F. Kerber for providing me with observations from the Magellan telescope. I acknowledge financial support by the Austrian Science Fund (FWF) under project P15065.

\section{References}

Leitherer, C. et al. 1999, ApJS, 123, 3

Mendes de Oliveira, C., Plana, H., Amram, P., Balkowski, C., \& Bolte, M. 2001, AJ, 121, 2524

Temporin, S., Weinberger, R., Galaz, G., \& Kerber, F. 2003a, ApJ, 584, 239

Temporin, S., Weinberger, R., Galaz, G., \& Kerber, F. 2003b, ApJ, 587, 671 